\title{
Design of Antenna Automated Measurement System
}

\author{
Chonghui Wang, Jingjian Huang, Lierong Wu, Naichang Yuan \\ National University of Defense Technology, China \\ *wilson_nudt@163.com
}

\begin{abstract}
Keywords: antenna; automated measurement system; dual-polarized; multi-channel; VC++
\end{abstract}
\begin{abstract}
In order to test and measure the dual-polarized, multi-channel antenna, a system was designed according to the actual working condition of the antenna. The hardware consists of the microwave network analyzer, three-dimensional turntable, switch matrix etc. The turntable is responsible for changing the antenna's direction. The switch matrix selects transmitter and receiver channels, and the network analyzer generates sweep signals and processes received signals. The software was designed by $\mathrm{VC}++$, it's flexible, easy to expand and upgrade.
\end{abstract}

\section{Introduction}

The technology of wireless communication has gained rapid development these years. To meet the higher and higher demand for communication, antenna technology is also bringing about new changes. In order to develop high performance antenna, a high-precision and high-efficiency test system could be extremely important to improve the quality of the antenna, shorten time-to-market, advance development and production efficiency of the antenna. The invention and widely application of virtual instrument has made it possible to test and measure automatically. Nowadays, the antenna automated measurement system has been widely used, and the measurement technology has become more sophisticated.

To ensure the system support the measurement of dual-polarized signal transmits antenna and multi-channel signal receiving antenna, the design of switch matrix was added to change the transmit and receive channels on the basis of microwave network analyzer and three-dimensional turntable. The corresponding software system is developed as well. The software not only be able to complete the test task, but also depends less on other tools, adopts module design method, all these bring convenience for system hardware expansion and upgrade in the future.

\section{Hardware Design}

The whole system mainly contains these hardware: Agilent N5230C 2-Port PNA-L microwave network analyzer, master computer, three-dimensional turntable, MPC08SP motion control card and turntable control computer, Agilent 83017A microwave amplifier, dual-polarized antenna and multichannel antenna, two switch matrix.

Among them, the N5230C microwave network analyzer has a frequency range of $10 \mathrm{MHz}$ to $40 \mathrm{GHz}$, provides two ports with two built-in sources. It can achieve $110 \mathrm{~dB}$ system and $122 \mathrm{~dB}$ receiver dynamic range, measure up to 32001 points, set up 32 measurement channels at most, and its measurement speed is 4 to $9 \mu$ s per point.

Three-dimensional turntable is composed of the inner ring, central and outer ring. The outer ring realizes azimuth movement, ranging from -170 degrees to +170 degrees; the central implementation of pitching motion, ranging from -90 degrees to +90 degrees; and inner ring can undertake 360-degree rolling movement. All of them are motor-driven.

The transmitting end is a dual-polarized antenna while the receiving end is a multi-channel antenna. Switch matrix is composed of power switch, microwave power divider, serial communication and Ethernet module. Signals enter at the input port, and be divided into equal parts, which part output is 
up to the commands received from serial port or network port. In practical, the signal of each channel is received at the same time.

The Agilent 83017A's frequency range is from $50 \mathrm{MHz}$ to $26.5 \mathrm{GHz}$, minimum gain is $23.5 \mathrm{~dB}$, maximum output power is $100 \mathrm{~mW}$, noise figure is $8.5 \mathrm{~dB}$ within 1.0 to $12.5 \mathrm{GHz}$, and gain flatness is $4.5 \mathrm{~dB}$ within 1.0 to $26.5 \mathrm{GHz}$.

The hardware connection of the system is shown in Fig. 1.

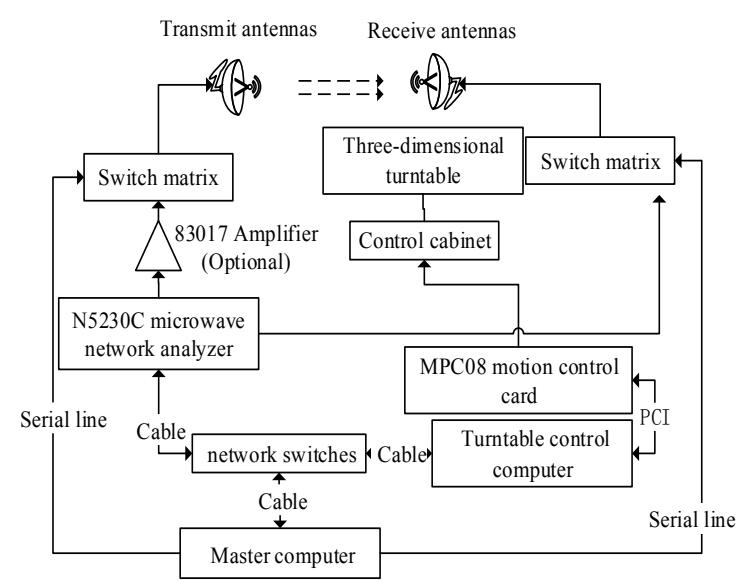

Figure 1. The connection diagram of the hardware system.

\section{Software Design}

The design goal of this software is to run fast and stable, be humanized, and dependent less.

\subsection{Tool Selection.}

The software chose Visual Studio 2010 as the software development platform. The Agilent IO Libraries Suite 16.2 was also selected because it contained VISA driver that essential for master computer to communicate with network analyzer. It also had Agilent Standard Instrument Control Library (SICL), VISA for the Common Object Model (COM), Agilent Connection Expert inside. SCPI was chosen as the control command. Although COM executes faster most of the time and is generally easier to be used, using COM can add additional complexity.

Drawing using GDI+ technology provided by Windows. It can draw lines, curves, graphs and images, and fully satisfy the project needs.

\subsection{Workflow.}

It's required to connect to $\mathrm{N} 5230 \mathrm{C}$ and the turntable control computer first, then set the three-dimensional turntable parameters and the network analyzer scanning parameters after the connection is successful. Once the measurement begins the turntable move to the start position, next the network analyzer carries out its first sweep, software processes and stores sweep results. When the data processing is completed, the program sends the next motion command. These procedures will be repeated until arriving at the destination. The flowchart is shown in Fig. 2.

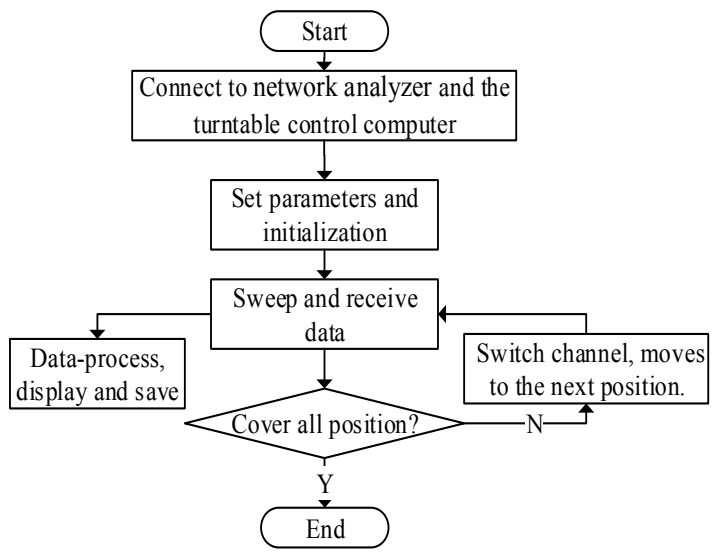

Figure 2. The measurement workflow 


\subsection{Function of Each Module}

Device connection. The connection between master computer and turntable control computer bases on the TCP/IP protocol, and master computer can communicate with the network analyzer through either LAN or GPIB. The IP address and port number (the network analyzer default is 5025) are required in network connections, while the GPIB device number and port number are required in GPIB connections. Whether the connection is successful or not will be prompted.

Turntable control. Here users can control turntable to accomplish the single axis rotation, two or three axes linkage operation remotely. From the feedback information, the program is able to judge turntable's running status and get the position of three axes.

Measurement setup. Measurement settings include network analyzer settings, turntable rotation settings, channel settings, and data storage settings, etc.

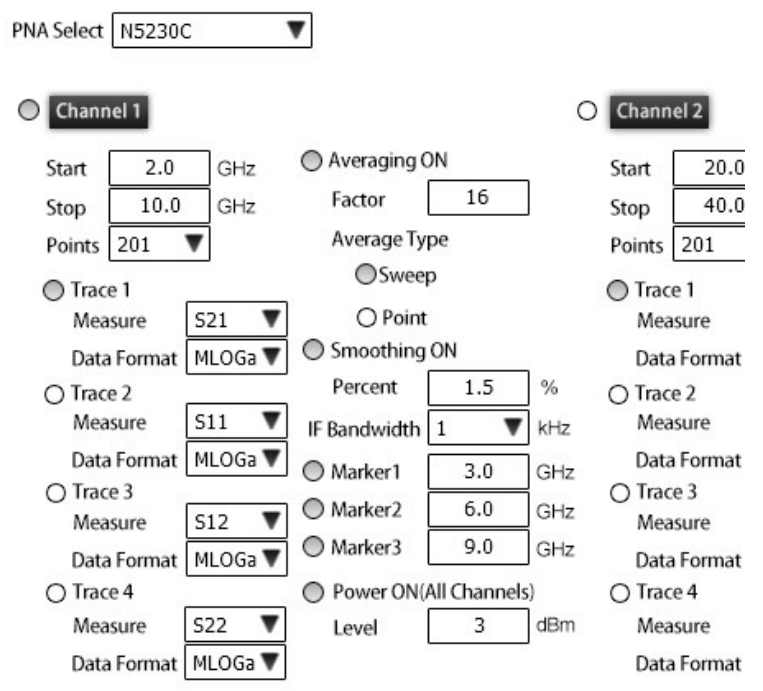

Figure 3. The network analyzer settings interface.

Network analyzer settings provide a model selection box that lists all the analyzer products for the users to choose on the basis of actual need. Functions are different among different network analyzers.

Turntable parameters settings include the number of each axis, start angle, end angle, step distance, rotation speed, moving priority, operation patterns, and enable state. The moving priority is three the rotation sequence of every axis that set during multi-axis motion. Operation patterns consist of continuous movement and stepping movement. The stepping pattern is more accurate because the network analyzer will not take the measure and receive data until the turntable moves into position. While the other pattern is time-saving for the measure is taken at same time intervals.

Channel settings consist of serial enabled state, port number, baud rate, and the channel count. The master computer sends commands to switch matrix through serial ports to control the transmission and receiving channels.

Data save settings include the selection of the data to get saved, the file name, and file path. The contents of the file include the network analyzer configuration information, polarization channel, receive channel, position of each axis and measurement data, etc. Because it takes some time to collect test results from the network analyzer, a new thread was created for receiving and saving data to guarantee the fluency of the interface operation.

Data display. Data display interface provides real-time display of the axis position, network analyzer channels and traces settings, progress of work and values at each frequency point under observation. The special points can be set at any time. If the input value is not on the list, the software will choose the closest point instead.

The software also creates a new thread for calculating reference coordinates. The traces change dynamically depends on the max and min values of the data are expected to display. 
Remote debug. Software is also available with a devices debug interface. Here users can send commands to network analyzer and turntable control computer directly, which is convenient to debug the hardware and software.

\section{Analysis of Test Results}

We carried out a simple test. In the measurement, the frequency range was from 2 to $10.0 \mathrm{GHz}$, IF bandwidth was $1 \mathrm{kHz}$, output power was $5 \mathrm{~dB}$, both the polarization channel and receiver channel number were 1 , the sweep range was from -70 degrees to 70 degrees in azimuth direction, the step distance was 1 degree. Four frequency points consisted of 2.0, 3.0, 6.0 and $9.0 \mathrm{GHz}$ were set for observation. The curves of the original data that received from the network analyzer and plotted by software are as follows:

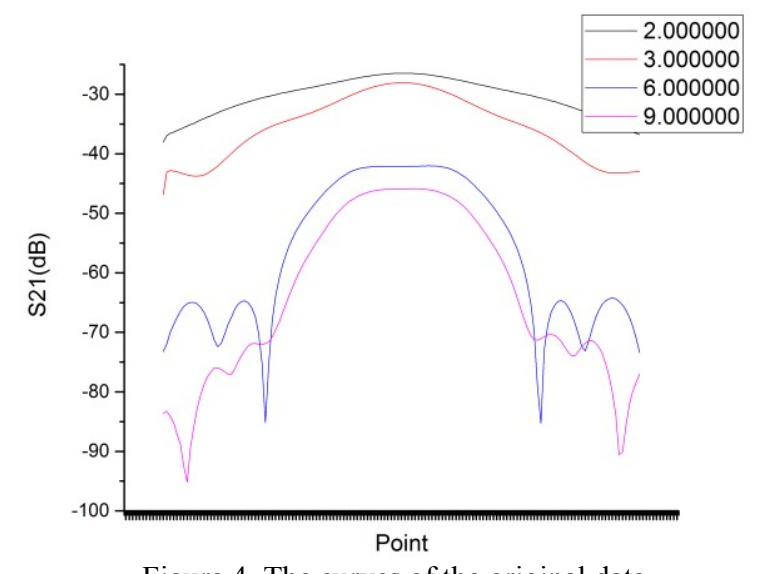

Figure 4. The curves of the original data.

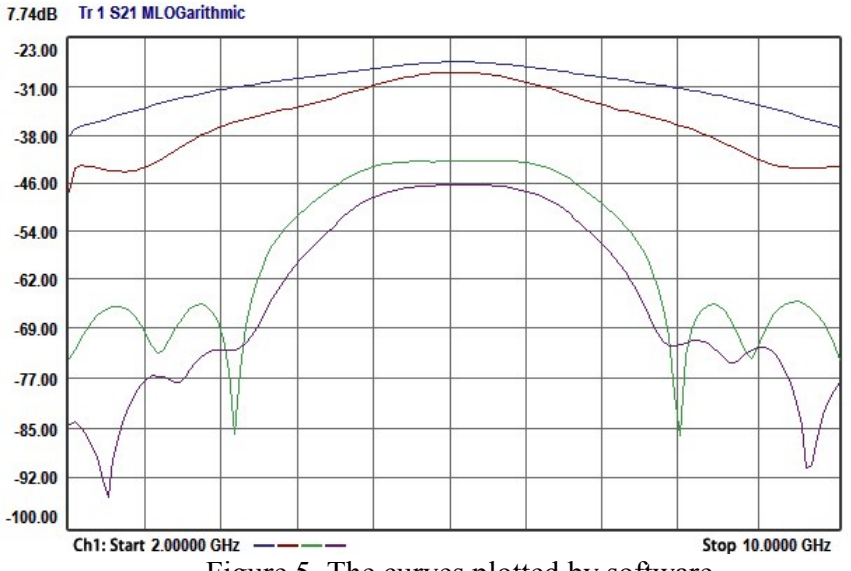

Figure 5. The curves plotted by software.

Comparing of results indicates that the software displayed curves were basically consistent with the ones of the original data. Thus, the software is able to show the changing trend of data correctly.

\section{Conclusions}

On the basis of the network analyzer and three-dimensional turntable, the system realized the measurement and test for dual-polarized antenna, multi-channel antenna by adding a switch matrix to the transmitting end and receiving end. The software takes full advantage of the instrument capabilities, saves the measure data in real-time, and the observed frequency values can be changed during the test process. The flexible use of multi-thread technology in data processing, dynamically allocating storage space according to the data size, ensure the efficient and stable operation of the software. At present, antenna automated measurement system has already been put into use. It can be used to measure multiple forms of active and passive antennas.

\section{References}

[1] L.N. Li, Visual C++ development technology, Tsinghua University Press, Beijing, 2010.

[2] Z.W. Zhuang, N.C. Yuan, Radar cross section measurement-Compact field theory and technology, National University of Defense Technology Press, Changsha, 2000.

[3] B.G. Liang, N.C. Yuan, J.P. Wang, Design of broadband RCS automatic test system, Computer Automated Measurement \& Control, 2004.12(1):64-65.

[4] Agilent technologies PNA series network analyzers printed version of PNA help 7.5, Agilent Technologies, Inc., 2007,

[5] Agilent VISA help with Agilent IO Libraries Suite revision 16.2, Agilent Technologies, Inc., 2012. 